\title{
Entangled Light Pulses from Single Cold Atoms
}

\author{
Giovanna Morigi, ${ }^{1}$ Jürgen Eschner, ${ }^{2}$ Stefano Mancini, ${ }^{3}$ and David Vitali ${ }^{3}$ \\ ${ }^{1}$ Grup d'Optica, Departament de Fisica, Universitat Autonoma de Barcelona, 08193 Bellaterra, Spain \\ ${ }^{2}$ ICFO_Institut de Ciències Fotòniques, 08860 Castelldefels (Barcelona), Spain \\ ${ }^{3}$ Dipartimento di Fisica, Università di Camerino, 62032 Camerino, Italy
}

(Received 15 July 2005; published 18 January 2006)

\begin{abstract}
The coherent interaction between a laser-driven single trapped atom and an optical high-finesse resonator allows one to produce entangled multiphoton light pulses on demand. The mechanism is based on the mechanical effect of light. The degree of entanglement can be controlled through the parameters of the laser excitation. Experimental realization of the scheme is within reach of current technology. A variation of the technique allows for controlled generation of entangled subsequent pulses, with the atomic motion serving as intermediate memory of the quantum state.
\end{abstract}

PACS numbers: 42.50.Dv, 03.67.Mn, 32.80.Qk

Coherently controlled atom-photon interfaces are the basic building blocks of implementations of quantum information processing and secure telecommunication with quantum optical systems. Experimental efforts towards this goal have reached several remarkable milestones. For instance, in the optical regime quantum correlations between atomic gases and light have been created and explored [1-4]; entanglement between a single trapped ion and a single photon has been demonstrated [5]; and highly controlled photonic interaction has been achieved with single atoms flying through resonators [6-9], or with atoms or ions trapped by an external potential inside an optical cavity [10-14]. The latter experiments have demonstrated, amongst others, the generation of Rabi oscillations between the atomic dipole and the cavity field $[7,13]$, laser action at the level of a single atom $[6,10]$, and the creation of single photons on demand $[9,11,14]$. In particular, in the experiment of Ref. [14] single-photon wave packets of adjustable shape and emission rate were generated. These realizations access novel regimes of engineering atomphoton interaction and open promising perspectives for implementing controlled nonlinear dynamics with quantum optical systems [15]. Besides its applications, this progress touches on interesting fundamental questions, such as how macroscopic nonlinear phenomena emerge from the dynamics of single quantum systems.

In this context, we show here that a single cold trapped atom in a high-finesse resonator can be used for the controlled, quantum-coherent generation of entangled light pulses, by exploiting the mechanical effects of atomphoton interaction. The atom's motional degrees of freedom act as a quantum medium that is used to establish entanglement between two field modes or to store and transmit quantum correlations between subsequent light pulses with variable delay. In all cases, at the end of the process the quantum medium is perfectly decorrelated from the electromagnetic field modes.

The specific application that we describe is that after a short coherent excitation pulse from the laser, the cavity will emit a pulse of two-mode squeezed, i.e., entangled, light. We also describe a variation of the scheme that allows for controlled generation of entangled subsequent pulses, with the atomic motion serving as intermediate memory of the quantum state.

Our scheme extends concepts developed for macroscopic oscillators [16] to a single quantum optical system, namely, to exploit the coupling between internal and external (motional) degrees of freedom for controlled, coherent creation of nonclassical light. While this coupling is extremely small in macroscopic systems, in atomic systems it is significant, thus moving its application for quantum information processing within experimental reach. Our study is also connected to ideas of mapping quantum states of atoms onto light inside a resonator [17,18], and to recent experimental and theoretical studies on quantum correlations in light scattering [1-4,19-21]. The proposal differs fundamentally from existing methods for generating pulsed squeezing [22] or intense pulses of polarizationentangled photons [23], which employ nonlinear crystals driven by a pulsed pump: in our case the microscopic nature of the medium allows for full coherent control of the light-matter quantum correlations and of the final quantum state of the generated light.

We first focus on the coherent generation of simultaneous, entangled light pulses. The physical system is sketched in Fig. 1. A single trapped atom of mass $M$, for example, an ion in an ion trap [12,13], is situated inside a high-finesse optical resonator and is driven by a laser. The atomic center-of-mass motion is a quantum harmonic oscillator of frequency $\nu$, described by the operators $b, b^{\dagger}$, which annihilate and create, respectively, a quantum of motional energy $\hbar \nu$ (a phonon). The atom's internal transition between states $|g\rangle$ and $|e\rangle$ has resonance frequency $\omega_{0}$ and linewidth $\gamma$ and interacts with a laser at frequency $\omega_{L}=\omega_{0}+\Delta$, where $\Delta$ is the detuning. The transition couples also with two modes of the resonator at frequencies $\omega_{1,2}=\omega_{L} \mp \nu$, which are described by annihilation and creation operators $a_{j}$ and $a_{j}^{\dagger}(j=1,2)$. These modes 


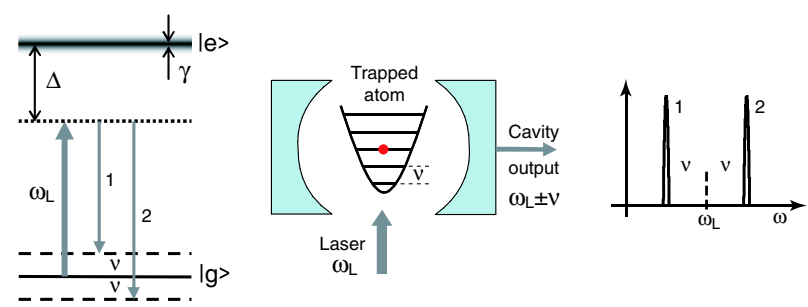

FIG. 1 (color online). Layout of the system and energy diagram. A single atom with internal energy levels $|g\rangle$ and $|e\rangle$ is confined by an external potential inside an optical resonator and is driven by a laser. The orientation of the vibrational mode under consideration has a nonzero projection onto the laser direction. The harmonic motion modulates the laser frequency, $\omega_{L}$, and the Stokes and anti-Stokes components at $\omega_{L} \pm \nu$ are resonant with two cavity modes, labeled 1 and 2 . The linewidth of $|e\rangle$ is $\gamma$, and $\Delta$ is the detuning between laser and atom.

can be, e.g., nondegenerate polarization modes split by $2 \nu$. Assuming that the cavity and the laser are sufficiently far off resonance with the atomic transition, $|\Delta| \gg \nu, \gamma$, the atom's internal degrees of freedom can be eliminated from the dynamics, and coherent Raman transitions are driven between the quantum motion and the cavity modes. They are described by the effective Hamiltonian $H=H_{1}+H_{2}$ with

$$
\begin{aligned}
& H_{1}=\mathrm{i} \hbar \chi_{1} a_{1}^{\dagger} b^{\dagger}+\text { H.c. } \\
& H_{2}=\mathrm{i} \hbar \chi_{2} a_{2}^{\dagger} b+\text { H.c. }
\end{aligned}
$$

Hamiltonian $H_{1}$ describes simultaneous creation or annihilation of a photon in mode 1 and a phonon, while $\mathrm{H}_{2}$ describes the exchange of excitation quanta between mode 2 and the motion. The coupling constants for these interactions are

$$
\begin{aligned}
& \chi_{1}=\eta g_{1}^{*} \Omega\left(\frac{\cos \theta_{L}}{\Delta-\nu+\mathrm{i} \gamma / 2}-\frac{\alpha_{1} \cos \theta_{c}}{\Delta+\mathrm{i} \gamma / 2}\right), \\
& \chi_{2}=\eta g_{2}^{*} \Omega\left(\frac{\cos \theta_{L}}{\Delta+\nu+\mathrm{i} \gamma / 2}-\frac{\alpha_{2} \cos \theta_{c}}{\Delta+\mathrm{i} \gamma / 2}\right),
\end{aligned}
$$

where $g_{1}, g_{2}$ are the vacuum Rabi frequencies of the cavity modes, $\Omega$ is the Rabi frequency of the laser, and $\eta=$ $\sqrt{\hbar k^{2} / 2 M \nu}$ is the Lamb-Dicke parameter [24], for which we assume $\eta \ll 1$, as typical for ion traps [25]. The geometric terms add up coherently the mechanical effects of laser and resonator [26], whereby $\theta_{L}$ and $\theta_{c}$ are the angles between the trap axis and the wave vectors $\vec{k}_{L}$ and $\vec{k}_{c}$ of the laser and the cavity, respectively $\left(\left|\vec{k}_{L}\right| \approx\left|\vec{k}_{c}\right|=k\right)$; the scalars $\alpha_{1}, \alpha_{2}$ depend on the field gradients of the cavity modes at the trap center. We exploit the asymmetry between $\chi_{1}$ and $\chi_{2}$ that originates from the different detunings, $\Delta \mp \nu$, of the two resonator modes from the atomic resonance. On a time scale on which the dynamics are coherent, this asymmetry is relevant if $\nu \gg \gamma$.
Let us now assume that a laser pulse of length $T$ interacts with the atom, whereby $\nu T \gg 1$ in order to ensure spectral resolution of the two cavity modes. We further assume that during this interaction incoherent processes such as spontaneous emission, cavity decay, and dephasing of the center-of-mass motion can be neglected. The appropriate parameter regime where these assumptions hold is discussed below. In this limit the dynamics are coherent and described by Eqs. (1) and (2). For $\left|\chi_{2}\right|>\left|\chi_{1}\right|$ the solution of the corresponding Heisenberg equations are periodic with frequency $\Theta=\sqrt{\left|\chi_{2}\right|^{2}-\left|\chi_{1}\right|^{2}}$ [16]. In particular, after a period $T_{\pi}=\pi / \Theta$ the two cavity modes exhibit quantum correlations, namely

$$
\begin{aligned}
& a_{1}\left(T_{\pi}\right)=\frac{\left|\chi_{2}\right|^{2}+\left|\chi_{1}\right|^{2}}{\Theta^{2}} a_{1}(0)-2 \frac{\chi_{1} \chi_{2}}{\Theta^{2}} a_{2}^{\dagger}(0), \\
& a_{2}\left(T_{\pi}\right)=\frac{2 \chi_{1} \chi_{2}}{\Theta^{2}} a_{1}^{\dagger}(0)-\frac{\left|\chi_{1}\right|^{2}+\left|\chi_{2}\right|^{2}}{\Theta^{2}} a_{2}(0) .
\end{aligned}
$$

Moreover, the two modes are decoupled from the centerof-mass oscillator, since $b\left(T_{\pi}\right)=-b(0)$. This solution implies that if at $t=0$ the state of the system is $\rho(0)=$ $\mu \otimes|0,0\rangle\langle 0,0|$, where $|0,0\rangle$ is the vacuum state for both cavity modes and $\mu$ is any state of the center-of-mass oscillator (for instance, a thermal distribution), then at $t=$ $T_{\pi}$ the state of the system is $\rho\left(T_{\pi}\right)=\mu^{\prime} \otimes|\psi\rangle\langle\psi|$, where

$$
|\psi\rangle=\left(\frac{1-r^{2}}{1+r^{2}}\right) \sum_{n=0}^{\infty}\left[-\frac{2 r}{1+r^{2}} e^{i \beta}\right]^{n}|n, n\rangle
$$

is a two-mode squeezed state of the two cavity modes, exhibiting EPR entanglement $[27,28]$. In Eq. (7) the parameters are $r=\left|\chi_{2} / \chi_{1}\right|$ and $\beta=\arg \left(\chi_{1}\right)+\arg \left(\chi_{2}\right)$, and $|n, n\rangle$ describes a state with $n$ photons in each cavity mode. The average number of photons per mode is $\langle n\rangle=$ $4 r^{2} /\left(1-r^{2}\right)^{2}$. Hence, if the laser pulse has duration $T_{\pi}$, after the interaction the cavity modes are entangled with one another and decorrelated from the quantum motion. The motion plays a fundamental role in establishing the entanglement; nevertheless, its initial state does not affect the efficiency of the process.

Equation (7) describes the field inside the cavity at the end of the laser interaction, provided that $\kappa T_{\pi} \ll 1$; i.e., the laser pulse is much shorter than the cavity lifetime $1 / \kappa$. This field is subsequently emitted from the cavity and contributes to the total cavity output field with a source term

$$
\mathbf{E}_{s}=\sum_{j=1,2} \vec{E}_{0 j} a_{j}(t)+\text { H.c. }
$$

where $a_{j}(t) \approx a_{j}\left(T_{\pi}\right) e^{-\kappa t}$ for $t>0$ [29]. Hence, $\mathbf{E}_{s}$ describes a bichromatic pulse that exhibits two-mode squeezing. The total output field is $\mathbf{E}_{\text {out }}=\mathbf{E}_{s}+\mathbf{E}_{f}$, where $\mathbf{E}_{f}$ is the free field contribution, i.e., the external vacuum modes mixing with $\mathbf{E}_{s}$ at the cavity output mirror. The total output 
field exhibits the two-mode squeezing of the source field over a time scale of $1 / \kappa$, provided that $\langle n\rangle \gg 1$.

Analogously, the atomic motion can mediate quantum correlations between subsequent pulses. A pair of entangled pulses with finite, variable delay between them can be obtained by coupling the atom to a single cavity mode at frequency $\omega_{c}$. In a first step, a laser pulse tuned to $\omega_{c}+\nu$ drives the atomic dynamics according to Hamiltonian $H_{1}$ [Eq. (1)] for a chosen interval of time. At the end of the pulse the motion and the cavity mode are in a two-mode squeezed state. After a delay $T_{12} \gg 1 / \kappa$, when the first light pulse has left the cavity, a second laser pulse at frequency $\omega_{c}-\nu$ drives the atom according to $\mathrm{H}_{2}$ [Eq. (2)]. With the appropriate pulse area, the previously created quantum state of the motion will be transferred to the cavity mode, such that the motion is decorrelated from the field modes, and the second pulse leaving the cavity exhibits correlated amplitude fluctuations with the first one. Hence the motion acts as an intermediate memory for the quantum state of the first pulse and mediates its entanglement with the second one, with the memory time limited only by the decoherence time of the ion motion, which can be extremely long.

A generic experimental setup to measure the entanglement of simultaneous pulses is shown in Fig. 2. The twomode output field $\mathbf{E}_{\text {out }}$ is split by a polarizing beam splitter, and the fluctuations of both modes are measured by balanced homodyne detectors [27], using local oscillators $E_{1,2}^{(\mathrm{LO})}$ with phases $\theta_{1,2}$. We define the corresponding quadratures of the two modes $j=1,2, Q_{j}\left(\theta_{j}\right)=q_{j}+\delta q_{j}$, where $q_{j}=a_{j} e^{i \theta_{j}}+a_{j}^{\dagger} e^{-i \theta_{j}}$ are the quadratures of the source fields and $\delta x_{j}$ denote the corresponding quadratures of the free fields. The measured currents at the detectors are $i_{j}(t)=c\left|E_{j}^{(\mathrm{LO})}\right| Q_{j}$, where $c$ is a scaling parameter assumed to be equal for the two modes. Time $t$, starting after the laser pulse, is considered to be defined on a grid $\delta t$, such that $\kappa \delta t \ll 1$; i.e., fluctuations are recorded on a time scale much faster than the cavity decay time [30]. The correla-

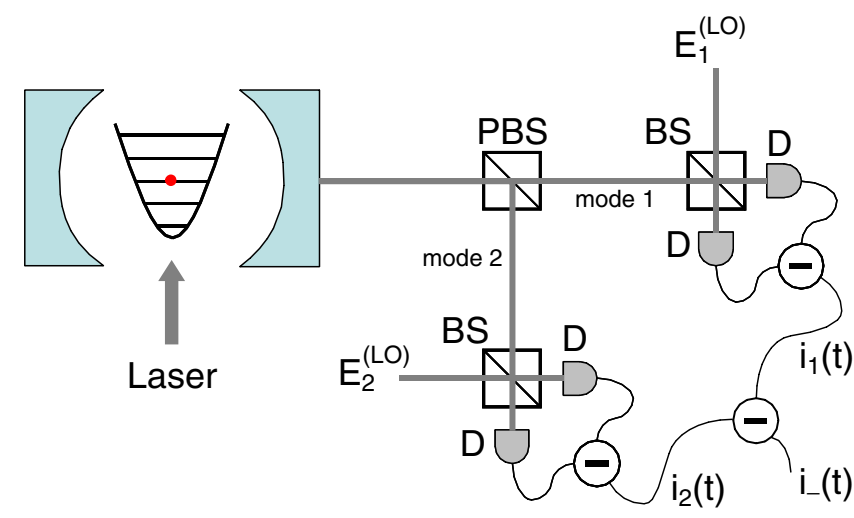

FIG. 2 (color online). Schematic setup for measuring relative fluctuations between the two electromagnetic field modes at the cavity output. PBS stands for polarizing beam splitter, BS for beam splitter, and D for detector. tions are measured through the difference current $i_{-}(t)=$ $i_{1}-i_{2}=c\left|E^{(\mathrm{LO})}\right|\left(Q_{1}-Q_{2}\right)$, where we have set the two local oscillator amplitudes equal. The current fluctuations at time $t$ are $\left\langle i_{-}(t)^{2}\right\rangle \propto C(t)$, where

$$
C(t)=1-\frac{\mathcal{R}(t)}{1+\mathcal{R}(t)} \frac{\left\langle q_{1} q_{2}\right\rangle}{\left\langle q_{1}^{2}\right\rangle+\left\langle q_{2}^{2}\right\rangle}
$$

with $\mathcal{R}(t)=\kappa \delta t \mathrm{e}^{-2 \kappa t}\left(\left\langle q_{1}^{2}\right\rangle+\left\langle q_{2}^{2}\right\rangle\right)$ and

$$
\begin{aligned}
& \left\langle q_{1}^{2}\right\rangle=\left\langle q_{2}^{2}\right\rangle=\left[\left(\left|\chi_{1}\right|^{2}+\left|\chi_{2}\right|^{2}\right)^{2}+4\left|\chi_{1} \chi_{2}\right|^{2}\right] / \Theta^{4}, \\
& \left\langle q_{1} q_{2}\right\rangle=\operatorname{Re}\left\{4 \chi_{1} \chi_{2}\left(\left|\chi_{1}\right|^{2}+\left|\chi_{2}\right|^{2}\right) e^{\mathrm{i}\left(\theta_{1}+\theta_{2}\right)} / \Theta^{4}\right\},
\end{aligned}
$$

and we have assumed that the free field is the coherent vacuum field. The value $C(t)=1$ corresponds to the shot noise limit for independent vacuum inputs into the homodyne detectors. The correlations $\delta\left(X_{1}-X_{2}\right)^{2}$ and $\delta\left(P_{1}+\right.$ $\left.P_{2}\right)^{2}$ of the two orthogonal quadratures $X$ and $P$ are obtained by setting $\theta_{1}=\theta_{2}=0$ and $\theta_{1}=-\theta_{2}=\pi / 2$, respectively, which leads to identical results for $C(t)$. Thus $C(t)<1$ is a signature (and, in fact, a quantitative measure [28]) of EPR-type entanglement.

Figure 3 shows the signal $C(t)$ for different values of the squeezing parameter $r$. A reduction below $10 \%$ of the shot noise level is reached on a time scale of $1 / \kappa$ for $r=1.1$. About 110 photons per mode are created in this case. It should be noted that for $r$ close to 1 , significant two-mode squeezing is observed over several cavity decay times, before the quantum noise level $C(t)=1$ is approached when the number of photons remaining in the cavity reaches the order of 1 .

We now discuss the parameter regime in which our description holds. Hamiltonians $H_{1}$ and $H_{2}$ are valid at first order in the Lamb-Dicke expansion, when higherorder, off-resonant, and inelastic scattering processes are negligible. Elastic scattering processes have been traced

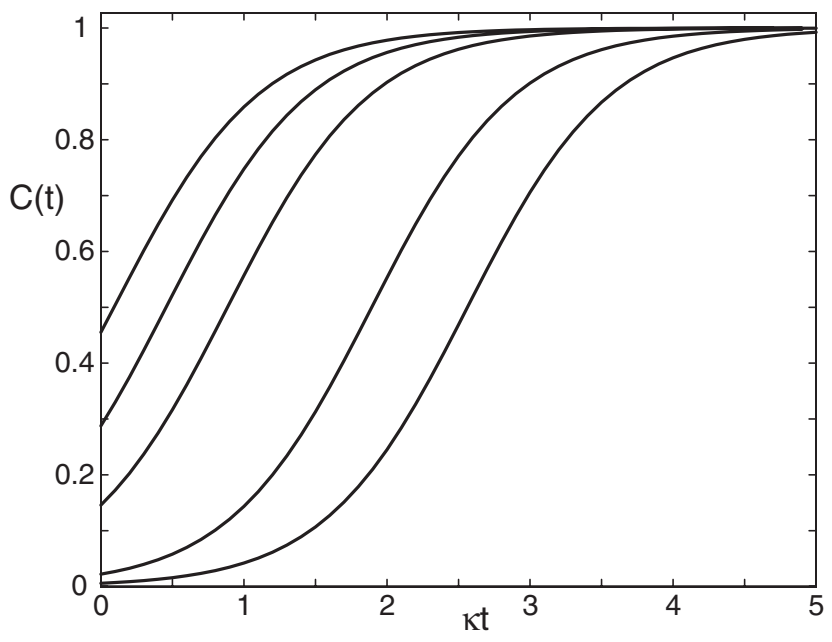

FIG. 3. Signal $C$ as a function of time, for $\theta_{1}+\theta_{2}=0$ and for values of the parameter $r=1.8,1.5,1.3,1.1,1.05$ (from left to right). A time resolution of $\delta t=\kappa / 10$ has been used. The other parameters are discussed in the text. 
out from these dynamics, since they do not affect the coherence properties of the center-of-mass oscillator and of the cavity modes [31]. Spontaneous scattering of cavity photons can be neglected if $\kappa \gg \gamma g_{j}^{2} / \Delta^{2}(j=1,2)$. The motion is not affected by spontaneous scattering provided that $\Theta \gg \eta^{2} \gamma \Omega^{2} / \Delta^{2}$. Cavity decay is negligible during the interaction if $\Theta \gg \kappa$. Moreover, Hamiltonians $H_{1}, H_{2}$ are based on the assumption that the cavity modes are spectrally resolved, i.e., $\nu \gg \Theta$. Therefore, one important inequality to be fulfilled is

$$
\nu \gg \Theta \gg \kappa .
$$

Finally, coherence of the center-of-mass oscillator during the whole time is a prerequisite for the efficiency of this scheme. We note that the parameter $r$, which determines the degree of entanglement, is controlled by the ratio $\nu / \Delta$, provided that $\nu \gg \gamma$, and by the ratio $g_{1} / g_{2}$, i.e., the values of the vacuum Rabi frequencies at the trap center [32].

The experimental conditions may, for example, be achieved on a $J=1 / 2 \leftrightarrow J^{\prime}=1 / 2$ or $F=0 \leftrightarrow F^{\prime}=1$ atomic transition with the quantization axis $\vec{B}$ along the cavity axis, and $\vec{B}, \vec{k}_{L}$, and laser polarization $\vec{E}_{L}$ mutually orthogonal. The parameter regime can be accessed by an indium ion which is confined by a trap at $\nu=2 \pi \times$ $3 \mathrm{MHz}$ and whose intercombination line at $\gamma=$ $2 \pi \times 360 \mathrm{kHz}$ is laser-driven with Rabi frequency $\Omega=$ $2 \pi \times 18 \mathrm{MHz}$ and detuning $\Delta=2 \pi \times 60 \mathrm{MHz}$. The ion couples to two nondegenerate polarization modes of a resonator of finesse $\mathcal{F}=10^{6}$ and free spectral range $\delta \omega=$ $2 \pi \times 1 \mathrm{GHz}$. With the resulting set of parameters $(g, \kappa, \gamma) / 2 \pi=(500,1,360) \mathrm{kHz}, \quad$ highly entangled pulses, characterized by $C(t)<0.1$, are observed on a time scale of the order of $0.1 \mathrm{msec}$. To obtain similar results with subsequent pulses requires a smaller Lamb-Dicke factor, i.e., a tighter trap. However, only a single cavity mode is needed, thereby relaxing several demanding conditions on the properties of the cavity and on the pulse duration, and thus allowing for a wider range of applications.

To conclude, we have shown that the motion of a single trapped atom inside an optical resonator can act as a quantum medium, which mediates entanglement on demand between simultaneous or subsequent radiation pulses. The process is based on the mechanical effect of light, which in the quantum regime allows for coherently controlling the interaction and thereby the degree of entanglement. It can be extended to the microwave regime by suitably driving atomic microwave transitions in a setup like the one discussed in [33]. In the future we will study correlations in the continuous-wave excitation of the ion, in the perspective of applications for quantum networking, like, for instance, discussed in $[34,35]$.

The authors gratefully acknowledge discussions with Christoph Becher, Markus Hennrich, and Scott Parkins.
This work was partly supported by the European Commission (CONQUEST network, MRTN-CT-2003505089, SCALA, Contract No. 015714); G. M. is supported by the Spanish Ministerio de Educación y Ciencia (Ramon-y-Cajal).

[1] C. H. van der Wal et al., Science 301, 196 (2003).

[2] A. Kuzmich et al., Nature (London) 423, 731 (2003).

[3] V. Josse et al., Phys. Rev. Lett. 91, 103601 (2003); V. Josse et al., Phys. Rev. Lett. 92, 123601 (2004).

[4] B. Julsgaard et al., Nature (London) 432, 482 (2004).

[5] B. Blinov et al., Nature (London) 428, 153 (2004).

[6] K. An et al., Phys. Rev. Lett. 73, 3375 (1994).

[7] J. M. Raimond et al., Rev. Mod. Phys. 73, 565 (2001); B. T. H. Varcoe et al., Nature (London) 403, 743 (2000).

[8] C. J. Hood et al., Science 287, 1447 (2000); P. W. H. Pinkse et al., Nature (London) 404, 365 (2000).

[9] A. Kuhn et al., Phys. Rev. Lett. 89, 067901 (2002); T. Legero et al., Phys. Rev. Lett. 93, 070503 (2004).

[10] J. McKeever et al., Nature (London) 425, 268 (2003).

[11] J. McKeever et al., Science 303, 1992 (2004).

[12] G. R. Guthöhrlein et al., Nature (London) 414, 49 (2001).

[13] A. B. Mundt et al., Phys. Rev. Lett. 89, 103001 (2002).

[14] M. Keller et al., Nature (London) 431, 1075 (2004).

[15] M. D. Lukin, Rev. Mod. Phys. 75, 457 (2003).

[16] S. Mancini et al., Phys. Rev. Lett. 90, 137901 (2003); S. Pirandola et al., Phys. Rev. A 68, 062317 (2003).

[17] H. Zeng and F. Lin, Phys. Rev. A 50, R3589 (1994); A. S. Parkins and H. J. Kimble, J. Opt. B 1, 496 (1999).

[18] A. Peng and A.S. Parkins, Phys. Rev. A 65, 062323 (2002).

[19] J. Ph. Karr et al., Phys. Rev. A 69, 063804 (2004).

[20] M. Jakob and J. Bergou, Phys. Rev. A 60, 4179 (1999).

[21] R. Guzmán et al., Phys. Rev. Lett. 96, 010502 (2006).

[22] M. E. Anderson et al., J. Opt. Soc. Am. B 14, 3180 (1997); G. S. Kanter et al., Opt. Express 10, 177 (2002).

[23] C. Simon and D. Bouwmeester, Phys. Rev. Lett. 91, 053601 (2003).

[24] S. Stenholm, Rev. Mod. Phys. 58, 699 (1986).

[25] The condition $\eta \ll 1$ can be significantly relaxed because, in the configuration we describe, only the first-order motional sideband couples to the cavity.

[26] J. I. Cirac et al., Phys. Rev. A 48, 2169 (1993); J. I. Cirac et al., Phys. Rev. A 51, 1650 (1995).

[27] M. D. Reid, Phys. Rev. A 40, 913 (1989).

[28] G. Giedke et al., Phys. Rev. Lett. 91, 107901 (2003).

[29] We assume that both modes decay at rate $\kappa$ and set the cavity output mirror at the spatial origin of the axis.

[30] The inverse of $\delta t$ corresponds to the spectral bandwidth over which vacuum fluctuations contribute to the signal.

[31] R. Ozeri et al., Phys. Rev. Lett. 95, 030403 (2005).

[32] If $\nu<\gamma$, very large values of $\Delta$ are required. In this case, $r$ can still be controlled by the ratio $g_{1} / g_{2}$.

[33] F. Mintert and Ch. Wunderlich, Phys. Rev. Lett. 87, 257904 (2001).

[34] J. I. Cirac et al., Phys. Rev. Lett. 78, 3221 (1997).

[35] B. Kraus and J.I. Cirac, Phys. Rev. Lett. 92, 013602 (2004). 